\title{
Consensus genetic linkage map construction and QTL mapping for plant height-related traits in linseed flax (Linum usitatissimum L.)
}

\author{
Jianping Zhang ${ }^{1 \dagger}$, Yan Long ${ }^{2 \dagger}$, Liming Wang ${ }^{1}$, Zhao Dang ${ }^{1}$, Tianbao Zhang ${ }^{2}$, Xiaxia Song ${ }^{2}$, Zhanhai Dang ${ }^{1 *}$ \\ and Xinwu Pei ${ }^{2^{*}}$
}

\begin{abstract}
Background: Flax is an important field crop that can be used for either oilseed or fiber production. Plant height and technical length are important characters for flax. For linseed flax, plants usually have a short technical length and plant height than those for fiber flax. As an important agronomical character for fiber and linseed flax, plant height is usually a selection target for breeding. However, because of limited technologies and methods available, there has been little research focused on discovering the molecular mechanism controlling plant height.

Results: In this study, two related recombinant inbred line (RIL) populations developed from crosses of linseed and fiber parents were developed and phenotyped for plant height and technical length in four environments. A consensus linkage map based on two RIL populations was constructed using SNP markers generated by genotyping by sequencing (GBS) technology. A total of 4497 single nucleotide polymorphism (SNP) markers were included on 15 linkage groups with an average marker density of one marker every $2.71 \mathrm{cM}$. Quantitative trait locus (QTL) mapping analysis was performed for plant height and technical length using the two populations. A total of 19 QTLs were identified for plant height and technical length. For the MH population, eight plant height QTLs and seven technical length QTLs were identified, five of which were common QTLs for both traits. For the PH population, six plant height and three technical length QTLs were identified. By comparing the QTLs and candidate gene information in the two population, two common QTLs and three candidate genes were discovered.
\end{abstract}

Conclusions: This study provides a foundation for map-based cloning of QTLs and marker-assisted selection for plant height-related traits in linseed and fiber flax.

Keywords: Flax, SNP, Linkage map, QTL, Plant height, Technical length

\section{Background}

Flax (Linum usitatissimum L.) is an annual self-pollinated diploid crop $(2 \mathrm{n}=2 \mathrm{x}=30)$ that can be used either for stem fiber or seed oil production about 7000 years ago [1]. Fiber flax and linseed flax are genetically the same but morphologically different [2]. One of the important differences between fiber and linseed flax is the character of

\footnotetext{
* Correspondence: 13669338239@163.com; Peixinwu@caas.cn
† Jianping Zhang and Yan Long contributed equally to this work.

* Correspondence: 13669338239@163.com; Peixinwu@caas.cn
†Jianping Zhang and Yan Long contributed equally to this work.

${ }^{1}$ Crop Institute, Gansu Academy of Agricultural Sciences, Lanzhou 730070, China

${ }^{2}$ Institute of Biotechnology, Chinese Academy of Agricultural Sciences, Beijing 100081, China
}

$\triangle B M C$

(c) The Author(s). 2018 Open Access This article is distributed under the terms of the Creative Commons Attribution 4.0 International License (http://creativecommons.org/licenses/by/4.0/), which permits unrestricted use, distribution, and reproduction in any medium, provided you give appropriate credit to the original author(s) and the source, provide a link to the Creative Commons license, and indicate if changes were made. The Creative Commons Public Domain Dedication waiver (http://creativecommons.org/publicdomain/zero/1.0/) applies to the data made available in this article, unless otherwise stated. fewer branches, while oil flax is usually about $70 \mathrm{~cm}$ high with many branches. Fiber flax is mainly grown in Northern Europe, Russia and China, and linseed flax is widely grown in Canada, India, America, Argentina and Germany, as well as Russia and China (Scientific Database of China Plant Species, http://db.kib.ac.cn). Flax oil contains a mixture of fatty acids, including saturated, monounsaturated and polyunsaturated fatty acids. Among the different types of fatty acids, the oil is rich in polyunsaturated fatty acids, particularly alpha-linolenic acid (ALA), the essential omega-3 fatty acid, and linoleic acid (LA), 
the essential omega- 6 fatty acid [3]. In China, linseed flax is mainly distributed in the Northwest and North areas, and has a planting history of about 2000 years [4].

As one of the important agronomical characters for both fiber and linseed flax, plant height is usually a selection target in breeding. Plant height trait has been found to have a significant co-relationship with seed yield traits, such as seed weight. For example, Contreras-Soto et al. found that some SNP markers on Chr19 controlled both the plant height and seed weight traits through a genome-wide association study (GWAS) in soybean [5]. Technical length is another selective breeding target in flax. In flax, Soto-Cerda et al. used 464 simple sequence repeat (SSR) markers to genotype 390 accessions and investigated the phenotypes of nine agronomic traits. Through GWAS analysis they discovered that 12 markers were significantly associated with six traits [6]. Halbauer et al.,(2017) used gSSRs to analyze 27 flax (Linum usitatissimum L.) accessions originating in the Alpine region and found a varying extent of accession-specific gene diversity (expected heterozygosity, HE) was revealed ranging from 0.05 to 0.51 [7]. However, there has been no research on plant height and technical length gene discovery in flax to date.

In conjunction with genomic tool development, a linkage map and QTL mapping are useful tools for discovering genes controlling important agronomic traits [8-10]. In flax, only a few linkage maps have been published. The earliest linkage map was constructed with 213 RAPD and RFLP markers in 18 linkage groups, and two Fusarium wilt QTLs were identified [11]. Later, Oh et al. also used RAPD and RFLP markers to construct a linkage map composed of 94 markers [12]. With the development of molecular markers, SSRs have become the major marker type for linkage map construction. Cloutier et al. constructed a linkage map that included 24 linkage groups with 113 EST-SSR markers for a DH population, and identified QTLs for seed color, linolenic acid content and linoleic acid content [13]. Then several maps were constructed based on SSR markers. For example, Cloutier et al. construct a consensus linkage map by combined three individual linkage maps incorporating 770 markers based on 371 shared markers including 114 that were shared by all three populations and 257 shared between any two populations [14]. Most of the QTL mapping analysis focused on fatty acid related traits, such as fatty acid composition, yield [15, 16], seed and flower color [17]. For example, Sudarshan et al.,(2017) identified a seed and flower color QTL "D" by QTL mapping analysis and identified a candidate gene [17].Today, SNP markers are the most efficient and abundant markers for mapping [18] and other applications, such as GWASs [19, 20], diversity analyses [21] and bulked segregate analysis [22].
Most SNP markers come from sequencing data generated by high-throughput sequencing technologies using genomic sequences as references. Whole genome sequences have been determined for a range of species, including soybean, Brassica rapa, cotton, flax and about other 60 plants (https://phytozome.jgi.doe.gov/pz/por tal.html). The flax genome includes about $302 \mathrm{Mb}$ of non-redundant sequence representing an estimated $81 \%$ genome coverage [23]. However, the sequence data have only been assembled into 88,384 scaffolds containing 43,384 genes, and these are not well anchored to a linkage map showing the relative positions of the genes. Recently, many new technologies have been developed for SNP marker discovery, such as genotyping-by-sequencing (GBS) $[24,25]$, restriction site-associated DNA (RAD) sequencing [26, 27], SLAF-seq (specific length amplified fragment sequencing) [28] and ddRAD sequencing [29]. In flax, GBS [30] and SLAF-seq [31] technologies have been used for SNP marker identification. Kumar et al.,(2012) constructed eight reduced representation libraries to do GBS and discovered 55,465 SNPs in the genome of flax [27] and then used 329 SNP and 362 SSR markers to construct a linkage map and identified a total of 20 QTLs corresponding to 14 traits. Yi et al.,(2017) used a F2 population to do SLAF-seq and construct a genetic map for flax and finally they discovered 4638 SNPs for genetic map construction. The final genetic map included 4145 SNP markers on 15 linkage groups and with $2632.94 \mathrm{cM}$ in length [31].

Thus, linkage map construction based on SNP markers has become easier and more efficient. Accordingly, candidate gene analysis for important agronomic traits has also become easier. For example, Singh et al. identified a candidate gene for 100-seed weight and root/total plant dry weight ratio under rainfed conditions in chickpea using whole genome resequencing and variant SNP loci collection [32]. Using recent tools for SNP marker identification, the aims of this study were to: 1) develop SNP linkage maps for two related recombinant inbred line (RIL) populations; 2) construct a consensus linkage map based on the two linkage maps; 3) perform QTL mapping for the plant height and technical length traits in the two related populations.

\section{Results}

Phenotype variation in the two populations

For the plant height and technical length phenotypes, the common male parent Heiya No.14 of the two populations showed higher values than both of the female parents, Macbeth and P.I.249991 (Fig. 1). The plant height and technical length values of the individuals in the two populations showed normal distributions in all of the environments (Fig. 2). The correlation analysis showed that the two traits in the two populations were 


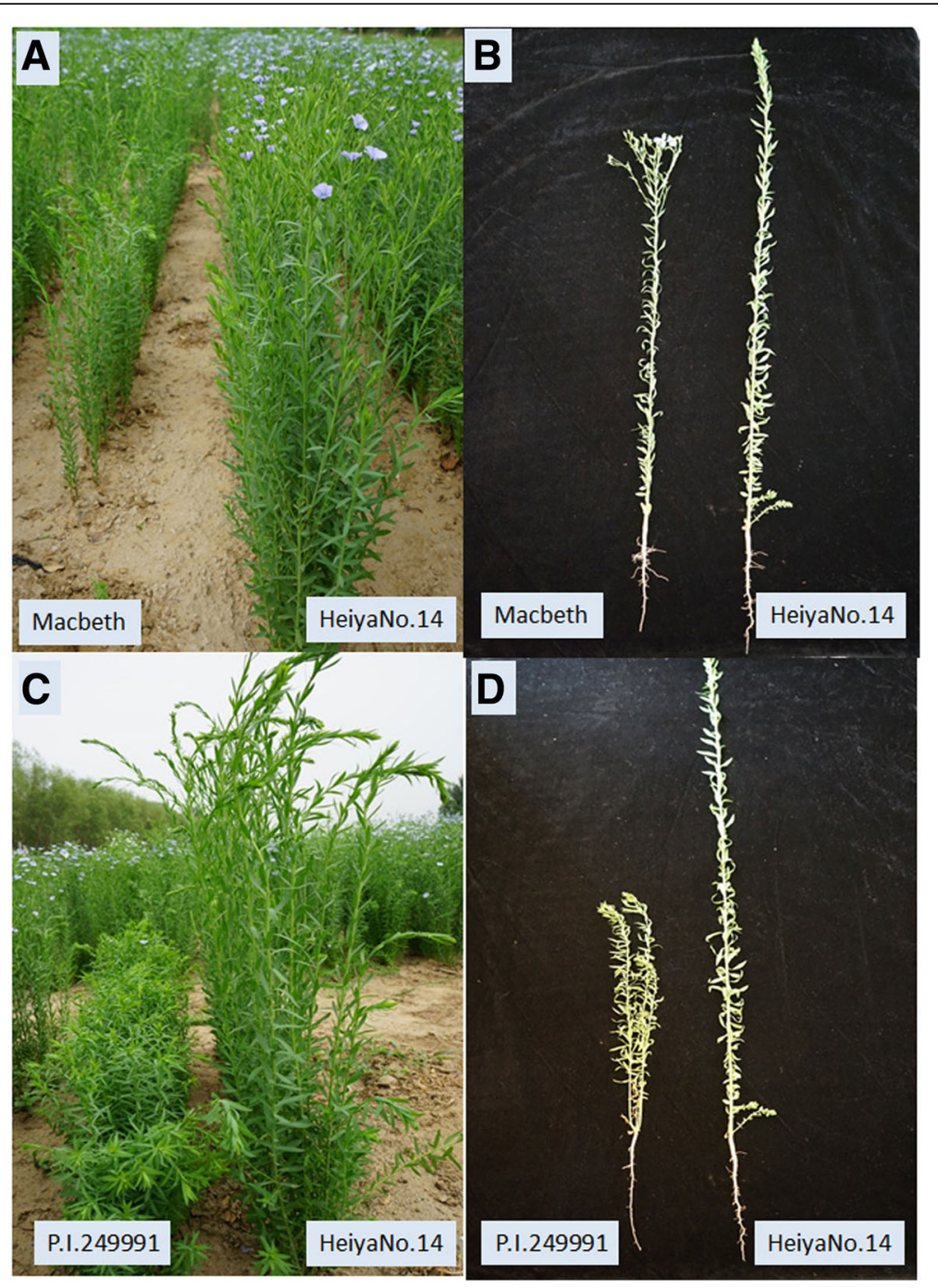

Fig. 1 Plant height phenotypes for the parents in the two populations. a, b Plant height phenotypes for the two parents, Macbeth and Heiya No.14, in the MH population; c, d plant height phenotypes for the two parents, P.I.294441 and Heiya No.14, in the PH population

positively related, and the correlation coefficient ranged from $0.7-0.85$ in different environments. The phenotypic variations of the two related populations were listed in Table 1. An interesting phenomenon was that, for plant height, the phenotypic values were lowest in Jingtai, followed by Lanzhou and Langfang, and highest in Yuanmou (Table 1, Fig. 2). The same tendency was observed for the technical length distribution in three of the environments.

\section{ddRADseq statistics for the two RIL populations}

The ddRAD seq protocol was used to construct sequence libraries for both the MH and PH RIL populations. The genomic DNA was double digested with the restriction enzymes $\mathrm{SacI}$ and $M s e \mathrm{I}$, and then fragments in a size range of 141-420 bp were recovered. Libraries from 12 different individuals tagged with 12 barcodes were pooled and sequenced on the Illumina HiSeq2000 platform. The three parents Macbeth, Heiya No.14 and P.I.249991 yielded 1.05(3.5× genome coverage), 2.59 (8.6x genome coverage) and $2.08(6.9 \times$ genome coverage) million PE reads, respectively. For the MH population, the 110 individuals yielded a total of 135.95 million $\mathrm{PE}$ reads, ranging from $0.38(1.3 \times$ genome coverage $)$ to 4.99 (16.6× genome coverage) million reads in different RILs with an average of $1.21(4.03 \times$ average genome coverage) million reads per RIL line (Fig. 3a). For the PH population, the 123 individuals yielded a total of 168.19 million PE reads, ranging from $0.31(1.03 \times$ genome coverage) to $5.9(19.7 \times$ genome coverage) million reads in different RILs with an average of 1.37 (4.57× average genome coverage) million reads per RIL line (Fig. 3b). 

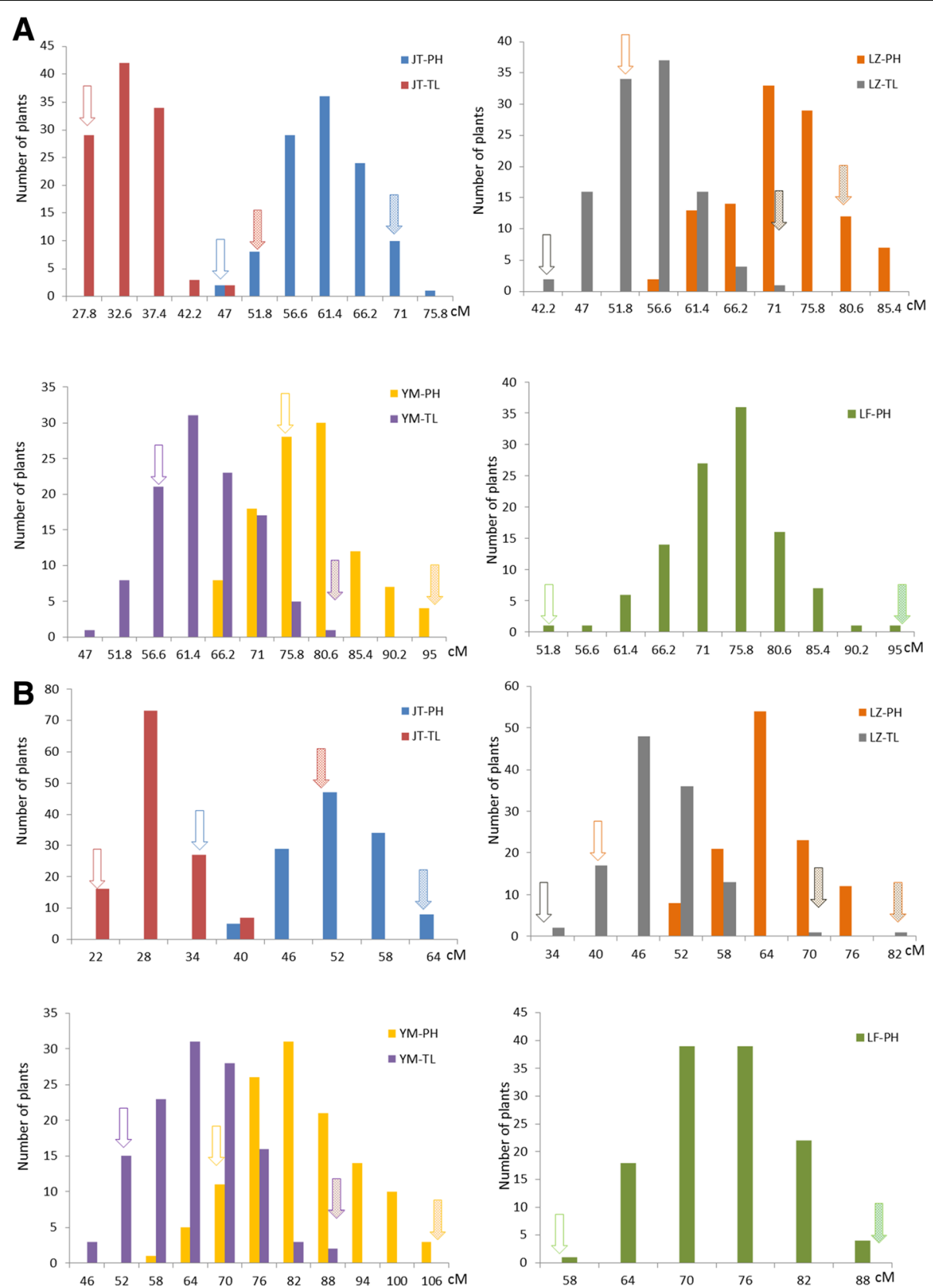

Fig. 2 Plant height and technical length distributions in different environments in the two populations. a PH distribution in the MH population. Solid arrows represent the female parent Macbeth, and arrows with oblique lines represent the male parent Heiya No.14. b PH distribution in the PH population Solid arrows represent the female parent Macbeth; arrows with oblique lines represent the male parent Heiya No.14. Solid arrows represent the female parent PI.29991, and arrows with oblique lines represent the male parent Heiya No.14

SNP genotyping and linkage map construction in the two RIL populations

After getting the raw sequencing data, three major steps were used to identify SNP markers. First, the $5 \mathrm{bp}$ index at the end of $5^{\prime}$ end was removed and the last 5 bp error-enriched nucleotides at the $3^{\prime}$ end were trimmed for each read. Therefore, $80 \mathrm{bp}$ PE reads were kept for the following analysis. Then, subsequent steps including recognizing the tags, constructing tag networks, removing erroneous tags and filtering out networks having only a single tag were done. Finally, 7399 and 5505 SNPs were identified in the $\mathrm{PH}$ and $\mathrm{MH}$ populations, respectively. The distribution of informative SNP numbers was 24.5 and 18.2 per megabase along the flax chromosome for each population. This mean that the polymorphic variation between Macbeth and Heiya No.14 was lower than the two parents in $\mathrm{PH}$ population. 
Table 1 Phenotypic variation of $\mathrm{MH}$ and $\mathrm{PH}$ population in four environments

\begin{tabular}{|c|c|c|c|c|c|c|c|c|c|c|}
\hline Trait & environments & Female $(\mathrm{cm})$ & Male (cm) & $\operatorname{Min}^{a}(\mathrm{~cm})$ & $\operatorname{Max}{ }^{b}(\mathrm{~cm})$ & $\operatorname{Mean}^{c}(\mathrm{~cm})$ & $S D^{d}$ & $C V(\%)^{e}$ & Kurto sis ${ }^{f}$ & Skewness $^{9}$ \\
\hline \multicolumn{11}{|c|}{ PH population } \\
\hline \multirow[t]{4}{*}{$\mathrm{PH}$} & LZ & 38.20 & 81.80 & 48.90 & 75.40 & 62.15 & 5.76 & 9.27 & 0.13 & -0.14 \\
\hline & JT & 29.65 & 63.68 & 35.50 & 62.65 & 49.98 & 5.46 & 10.92 & 0.03 & -0.52 \\
\hline & YM & 65.50 & 110.60 & 55.70 & 105.80 & 78.97 & 9.77 & 12.37 & 0.06 & -0.06 \\
\hline & LF & 47.67 & 103.56 & 50.78 & 85.00 & 69.87 & 6.51 & 9.32 & -0.24 & -0.16 \\
\hline \multirow[t]{3}{*}{$\mathrm{TL}$} & LZ & 27.50 & 70.30 & 31.70 & 81.20 & 56.45 & 5.99 & 10.62 & 1.64 & 0.40 \\
\hline & JT & 20.86 & 48.38 & 16.50 & 41.40 & 28.95 & 4.52 & 15.63 & 0.90 & 1.31 \\
\hline & YM & 51.32 & 88.30 & 38.60 & 128.00 & 83.30 & 10.39 & 12.47 & 1.63 & 0.30 \\
\hline \multicolumn{11}{|c|}{ MH population } \\
\hline \multirow[t]{4}{*}{$\mathrm{PH}$} & $L Z$ & 51.00 & 80.50 & 51.90 & 84.70 & 68.75 & 6.73 & 9.78 & 0.07 & -0.44 \\
\hline & JT & 49.40 & 70.75 & 43.30 & 82.00 & 58.53 & 5.99 & 10.23 & 0.57 & 0.89 \\
\hline & YM & 74.10 & 110.60 & 59.40 & 94.70 & 75.19 & 7.35 & 9.77 & 0.27 & -0.33 \\
\hline & LF & 50.90 & 93.50 & 50.78 & 94.78 & 70.01 & 7.89 & 11.26 & -0.11 & 0.02 \\
\hline \multirow[t]{3}{*}{$\mathrm{TL}$} & LZ & 41.87 & 68.90 & 35.40 & 67.00 & 51.09 & 5.82 & 11.39 & 0.05 & -0.36 \\
\hline & JT & 26.30 & 53.50 & 23.15 & 50.75 & 30.65 & 4.43 & 14.45 & 0.84 & 1.98 \\
\hline & YM & 55.10 & 80.00 & 46.40 & 75.90 & 60.04 & 6.70 & 11.16 & 0.07 & -0.58 \\
\hline
\end{tabular}

${ }^{a} \operatorname{Max}(\mathrm{cm})$ : The maximum value of phenotypic data in the two RIL populations

${ }^{\mathrm{b}} \mathrm{Min}(\mathrm{cm})$ : The Minimum value of phenotypic data in the two RIL populations

${ }^{c}$ Mean $(\mathrm{cm})$ : The average value of phenotypic data in the two RIL populations

${ }^{\mathrm{d}} \mathrm{SD}$ : Standard deviation of the phenotypic trait

${ }^{\mathrm{e}} \mathrm{CV}$ (\%): Coefficient of variation of the phenotypic trait

fSkewness: Skewness of the phenotypic trait

${ }^{9}$ Kurtosis: Kurtosis of the phenotypic trait

To construct the linkage maps, one SNP was selected from each allelic tag pair to represent the locus, which resulted in a set of 4348 and 3231 SNPs for the PH and $\mathrm{MH}$ population, respectively. After deleting the distorted SNP markers, finally a total of 2788 SNP markers distributed on 15 linkage groups were mapped for $\mathrm{PH}$ population, with a length of $1138 \mathrm{cM}$. The average marker density was 2.45 markers/cM. The LGs ranged from 15 to $137 \mathrm{cM}$ and contained 36 to 329 markers. For the PH map, a total of 2120 segregating markers were mapped on 15 linkage groups, and the total length was $1272 \mathrm{cM}$. There were 34 to 267 markers distributed on different LGs. The average marker density was 1.67 markers/cM. All the information for the SNP linkage maps is listed in Table 2. By combining the two individual linkage maps, an integrated linkage map was constructed. In total, the integrated map included 4497 molecular markers and was $1658 \mathrm{cM}$ long. For each LG, the marker number ranged from 154 to 576 (Table 2, Additional file 1, Additional file 2). The biggest linkage group was $L u 9$ with 451 integrated markers, while the smallest linkage group was $87 \mathrm{cM}$ and contained 154 molecular markers. Then the flanking sequences (Additional file 2) of all of the 4497 SNP markers were used for BLAST with the published flax genome. Totally, 1996 scaffolds were identified, and among them 1493 scaffolds were non-redundant. The other 2501 SNP markers couldn't be mapped to the reference genome, which mean that genetic differences existed in different flax cultivars.

\section{QTL mapping of plant height and technical length in the two RIL populations}

QTL mapping analysis was done using the WinQTLcart2.5 software. After initial QTL mapping analysis, several plant height and technical length QTLs were identified in different environments in the two populations (Table 3, Fig. 4). For the MH population, 10 QTLs distributed on eight linkage groups were identified, the phenotypic variation ranged from 18 to $26 \%$, and most of the additive effect was negative, which means that most of the alleles from the female parent Macbeth made the plants shorter. For the plant height trait, eight QTLs distributed in seven linkage groups were identified. Seven QTLs on six linkage groups were detected for the technical length trait. Comparing the QTLs of the two traits showed that five QTLs were shared by both traits, such as uq.C5-1 and uq.C6-1. Conversely, three unique QTLs were detected in multiple environments and the other seven QTLs were environment-specific. For the PH population, nine QTLs distributed on eight linkage groups were identified, and the phenotypic variation ranged from 18 to 68\% (Table 2, Fig. 4). Six QTLs distributed in six linkage 


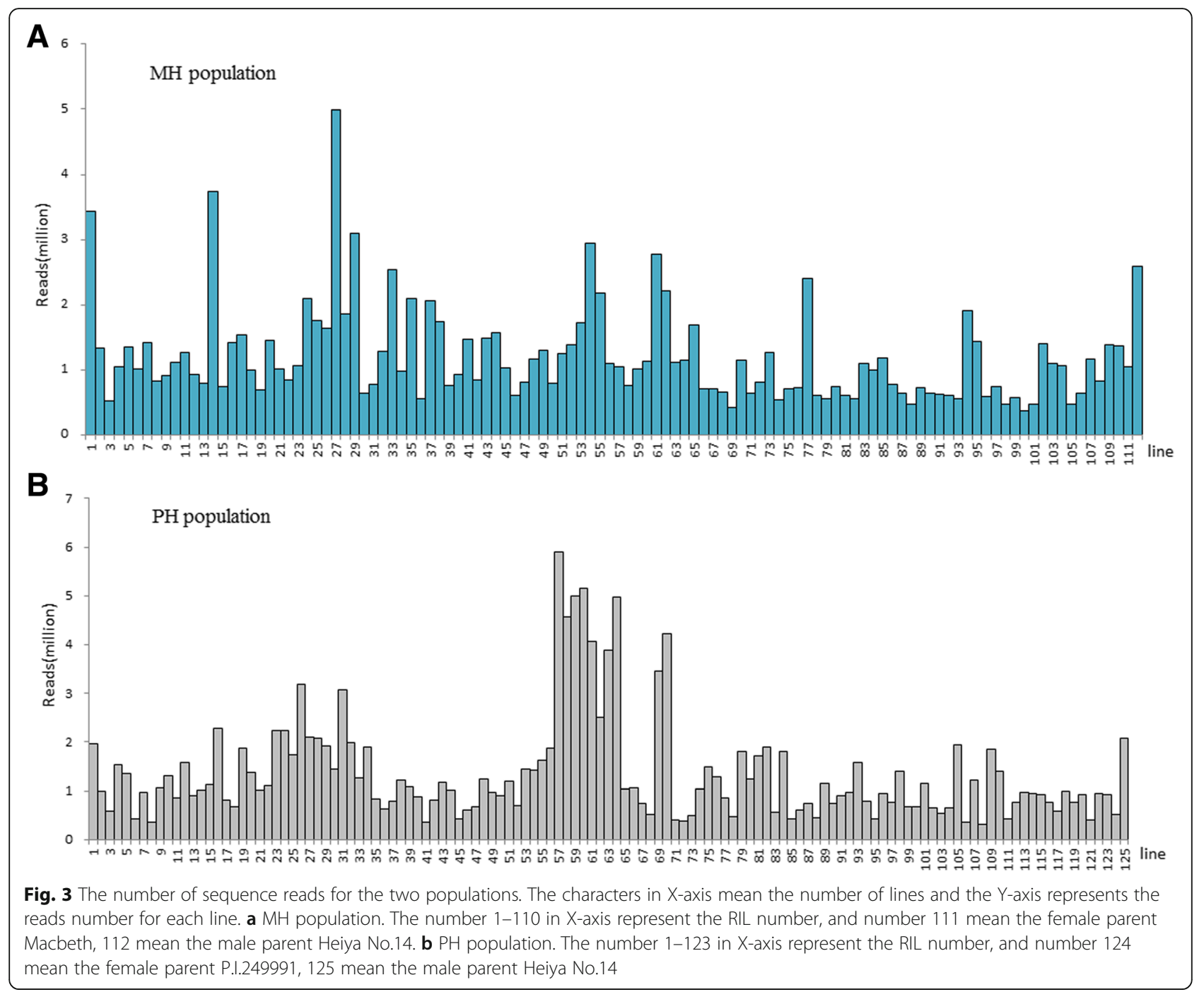

groups were identified for the plant height trait, and three QTLs on three linkage groups were detected for the technical length trait. Comparing the QTLs of the two traits showed that no QTLs controlled both traits. Additionally, only one unique QTL was detected in two environments; the other QTLs were environment-specific. When the QTL mapping results were compared between the two populations, only one QTL located in Lu11 was detected in both populations. After the QTL mapping analysis, the candidate genes of the QTLs were identified based on the sequence information of the SNP markers. In total, 28 potential flax candidate genes were located in the QTL confidence intervals, including 17 candidate genes for the $\mathrm{MH}$ population and 11 candidate genes for the $\mathrm{PH}$ population (Table 3). Gene annotation results showed that different gene functions were potentially involving in the plant developing in flax, such as LACCASE, MYB regulators. And the actual functional genes controlling the plant height and technical length QTLs would be discovered by further study.

\section{Discussion}

In the current study, a high-density integrated linkage map was constructed based on individual linkage maps from the $\mathrm{MH}$ and $\mathrm{PH}$ populations. Differing from previously published linkage maps in flax, the molecular markers used in this study were SNP markers based on the high-throughput sequencing technology-ddRAD sequencing method. Compared with traditional RAD sequencing, the ddRAD method simplifies the experimental process and reduces the cost of reduced representation library construction, and can be used in species without a reference genome [33]. Poland et al. first used this technology to develop thousands of SNP markers and constructed two high-density linkage maps for barley and wheat [34]. Subsequently, ddRAD-derived SNP linkage maps were constructed in different species, such as peanut [35] and strawberry [36]. In flax, the first SNP maps were developed by the GBS technology. Kumar et al., (2012) first used GBS technology to discovering SNPs among 8 
Table 2 Mapping statistics for the three individual and the consensus genetic maps of flax

\begin{tabular}{|c|c|c|c|c|c|c|c|c|c|}
\hline \multirow{2}{*}{$\begin{array}{l}\text { Linkage } \\
\text { group }\end{array}$} & \multicolumn{3}{|l|}{ MH map } & \multicolumn{3}{|l|}{ PH map } & \multicolumn{3}{|c|}{ Integrated } \\
\hline & $\begin{array}{l}\text { marker } \\
\text { number }\end{array}$ & $\begin{array}{l}\text { Length } \\
\text { (cM) }\end{array}$ & $\begin{array}{l}\text { average } \\
\text { distance(cM) }\end{array}$ & $\begin{array}{l}\text { marker } \\
\text { number }\end{array}$ & Length(cM) & $\begin{array}{l}\text { average } \\
\text { distance(cM) }\end{array}$ & $\begin{array}{l}\text { marker } \\
\text { number }\end{array}$ & Length(cM) & $\begin{array}{l}\text { average } \\
\text { distance(cM) }\end{array}$ \\
\hline Lu1 & 88 & 46 & 0.52 & 175 & 75 & 0.43 & 257 & 83 & 0.32 \\
\hline Lu2 & 36 & 15 & 0.42 & 204 & 119 & 0.58 & 238 & 124 & 0.52 \\
\hline Lu3 & 128 & 63 & 0.49 & 210 & 105 & 0.50 & 333 & 108 & 0.33 \\
\hline Lu4 & 143 & 67 & 0.47 & 37 & 57 & 1.55 & 177 & 74 & 0.42 \\
\hline Lu5 & 187 & 90 & 0.48 & 95 & 65 & 0.68 & 267 & 110 & 0.41 \\
\hline Lu6 & 213 & 72 & 0.34 & 148 & 52 & 0.35 & 334 & 81 & 0.24 \\
\hline LuT & 122 & 59 & 0.49 & 34 & 50 & 1.47 & 154 & 67 & 0.43 \\
\hline Lu8 & 329 & 104 & 0.32 & 267 & 141 & 0.53 & 576 & 167 & 0.29 \\
\hline Lu9 & 258 & 112 & 0.43 & 199 & 128 & 0.64 & 451 & 184 & 0.41 \\
\hline Lu10 & 184 & 69 & 0.38 & 90 & 54 & 0.60 & 261 & 111 & 0.43 \\
\hline Lu11 & 100 & 57 & 0.57 & 100 & 97 & 0.97 & 192 & 102 & 0.53 \\
\hline Lu12 & 159 & 53 & 0.34 & 148 & 65 & 0.44 & 298 & 83 & 0.28 \\
\hline Lu13 & 348 & 137 & 0.39 & 214 & 122 & 0.57 & 523 & 171 & 0.33 \\
\hline Lu14 & 240 & 91 & 0.38 & 40 & 58 & 1.45 & 277 & 107 & 0.39 \\
\hline Lu15 & 253 & 103 & 0.41 & 159 & 84 & 0.53 & 159 & 84 & 0.53 \\
\hline Total & 2788 & 1138 & 0.41 & 2120 & 1272 & 0.60 & 4497 & 1658 & 0.37 \\
\hline
\end{tabular}

flax cultivars [30], meanwhile Cloutier et al., (2012) constructed consensus linkage map by using these SNP markers and SSR markers, and finally there were 770 markers distributing in 15 linkage groups [14]. Until now, the latest linkage map published by Kumer et al.,(2015) was with 329 SNPs and 362SSRs. Compared with the previous linkage maps, the current SNP map generated in this study is the most high-density map to date and will help flax researchers to discover flax genes more easily.

Based on the two individual linkage maps, a consensus linkage map was constructed. Mapping of genetic markers using multiple populations provides increased genome coverage because it is unlikely that multiple parents would all be fixed (monomorphic) in the same genomic regions. In the $\mathrm{MH}$ linkage map, the $\mathrm{Lu} 2$ linkage group contained only 36 molecular markers and was $15 \mathrm{cM}$, whereas in the PH linkage map, there were 204 molecular markers in the Lu2 linkage group covering $119 \mathrm{cM}$. Thus, once these two linkage groups were integrated together, the coverage of this group was greatly increased. Additionally, we anchored the published scaffolds onto the consensus map based on the SNP sequencing information. In total, 1493 non-redundant scaffolds were mapped onto the linkage map, which means that these scaffolds were physically mapped onto the linkage map and will help to construct the whole physical map. While there were still 2501 SNP markers couldn't be anchored with published reference genome sequences. One of the reasons was genetic differences existing between the reference genome and the current flax cultivar genomes. The other possible reason was that the coverages of the reference genome published in 2012 [23] were not enough to dissect the whole flax genome. So the consensus linkage map would be benefit for flax genome assembly. Additionally, the flanking sequencing information could help to discover candidate genes for target QTLs of specific traits.

Plant height is an important agronomic trait affecting crop performance, particularly lodging and consequently yield and quality. Technical length is also an important trait for flax breeding. Previous studies have shown that plant height and technical length are positively correlated. In general, flax plants with a greater technical length also have a greater plant height. There are different demands for plant height and technical length in flax because of its different usages. Specifically, fiber-type flax needs to be taller, while linseed-type flax needs to be relatively short. Thus, different alleles that can enhance or reduce plant height and technical length phenotypes are needed according to different breeding purposes for flax. In this study, the phenotype of plant height showed obvious differences among the four environments. Additionally, the same phenotype distribution was found in three locations. The average value of plant height was smallest in Jingtai, followed by Lanzhou and Langfang, and largest in Yuanmou. This was because of the latitude, longitude and temperature differences in these locations. It was easy to understand why the plant height and technical length were highest in Yuanmou. We planted the two populations in October and the growth cycle was 5 months long, which was much longer than 


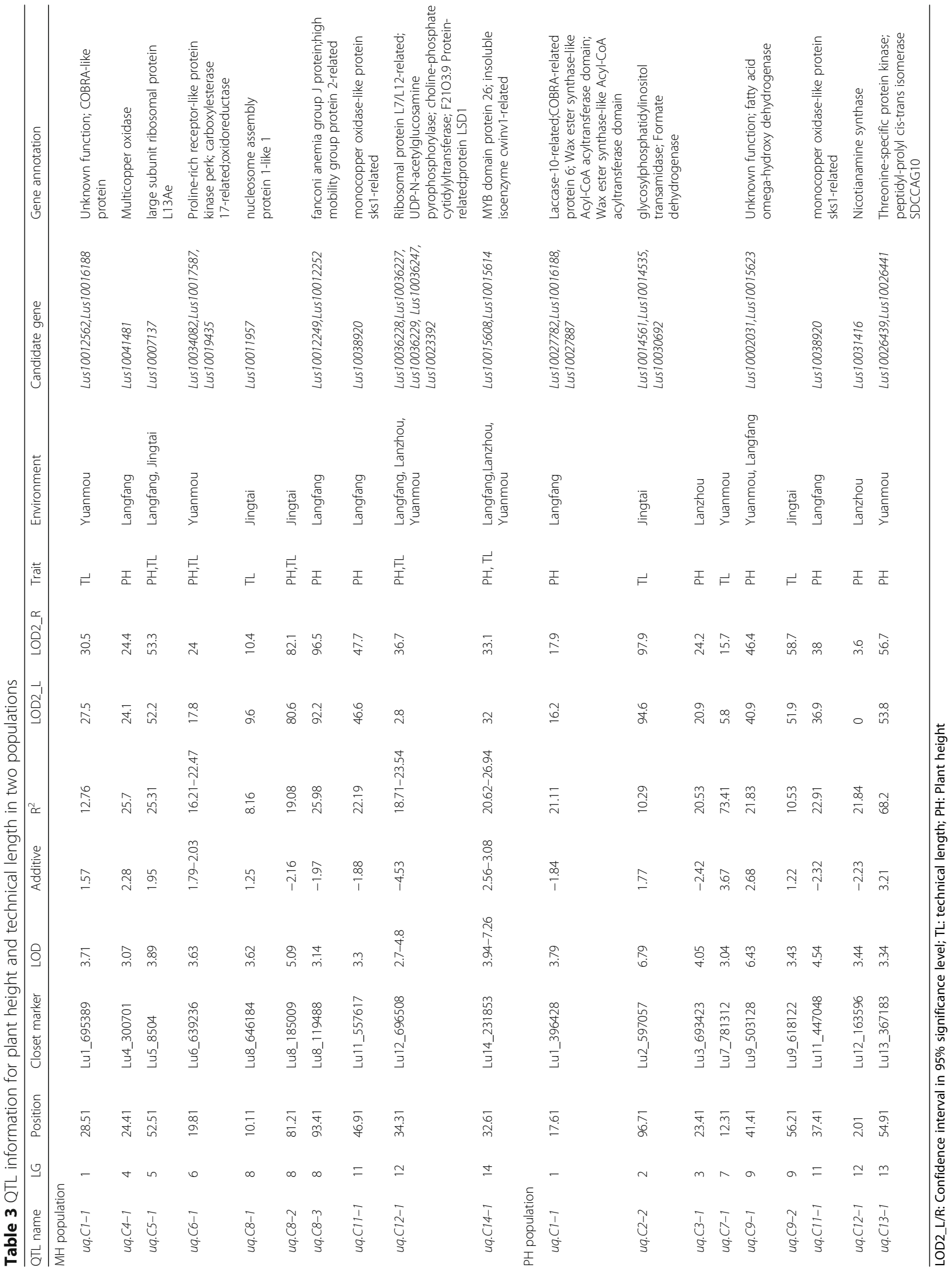




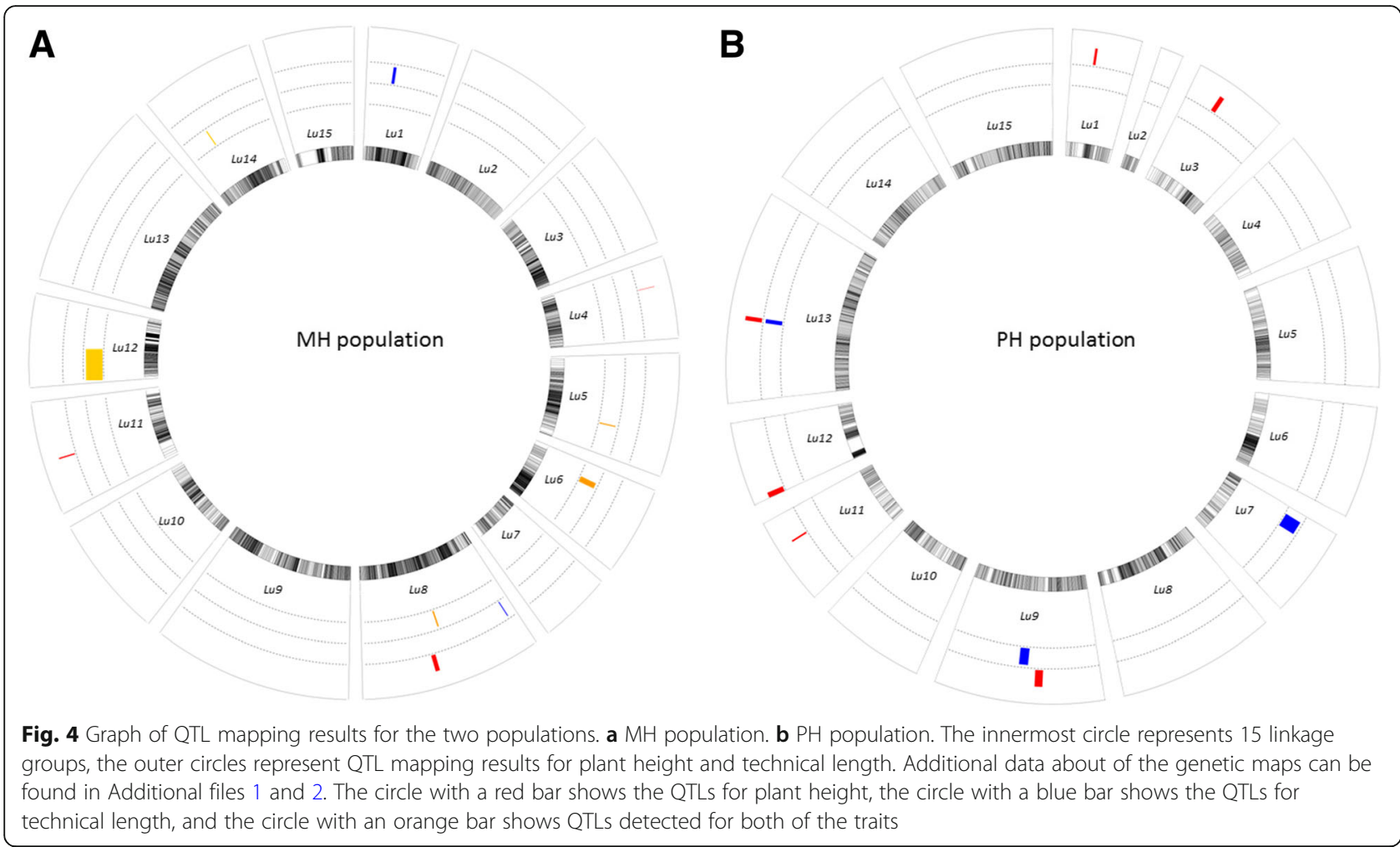

in the other three locations. In the other three locations, we planted the populations in April and harvested the seeds in early July, so the life cycle was only 3 months. Among these three locations, plant height was greatest in Langfang, followed by Lanzhou and lastly Jingtai, which means that plant height was positively correlated with longitude and negatively correlated with latitude. Several previous studies have shown that different environmental factors can influence plant height. For example, a photoperiod insensitive allele of the major photoperiod regulator Ppd-1, located on group 2 chromosomes, can have pleiotropic effects on plant height [37]. In flax, Soto-Cerda et al., (2014) used GWAS approach to identify genes relating to 9 agronomic traits including plant height. The results showed that one SSR marker was not only relate with flowering time, also relate with plant height [6]. We then compared the QTLs got from the GWAS analysis and from the current study, it was found that the QTLs were not same in the two studies. The reason was maybe the different genetic background of the research materials. Besides comparing the results from two different studies, we compared the QTL mapping results from the two populations. The results showed that most of the QTLs were unique, meaning that the QTLs were significantly influenced by the genetic background. One unique QTL uq.C11-1 located in Lu11 was detected in both populations, and the candidate gene Lus10038920 was identified. This showed that a common candidate gene contributed to the plant height trait in both of the populations. Thus, we could focus on this QTL and try to use the sequence information from the SNP markers for MAS in flax.

\section{Conclusions}

Flax is an important oilseed crop over the world, while until now there were few researches about gene and genomic information. In the current study, two RIL populations were used as materials to generate SNP markers and investigate the genetic control of plant height-related traits. As a result, a consensus high density linkage map was constructed based on the two individual linkage maps. Based on the linkage map and phenotypes, QTL mapping analysis was done for plant height and technical length. Totally there were 19 QTLs were identified. For MH population, eight plant height QTL and 7 technical length QTL were identified, and 5 are common QTLs. For PH population, there were 6 plant height QTL and 3 technical length QTL respectively. After comparing the QTL and candidate gene information of the two populations, two common QTLs and three candidate genes were discovered. This study provides the foundation for assisting in map-based cloning of the QTL and marker assisted selection of plant height related traits in linseed and potentially fibre flax. 


\section{Methods}

\section{Plant materials}

Two populations were used for linkage map construction and QTL mapping. The first was a Macbeth/Heiya No.14 (MH) RIL population. The MH RIL population RILs was developed from.

"Macbeth $\times$ Heiya No.14" by single-seed descent in seven generations. Macbeth is a Canadian 'conventional' oilseed-type cultivar with [38] 55-57\% linolenic acid, and Heiya No.14 is a Chinese fiber cultivar [39]. In total, 110 RILs were randomly selected from the original 235 lines and were used for genotyping and phenotyping analysis.

The second population was a P.I.249991/Heiya No.14 (PH) population, which consisted of 123 R7-derived RILs. The RILs were generated from a cross between the oilseed-type P.I.249991 with about 53\% linolenic acid [40] and the Chinese fiber flax variety Heiya No.14 by single-seed descent. As with the $\mathrm{MH}$ population, the plant height phenotypes of the two parents were significantly different (Fig. 1). The two populations were developed in Crop Institute of Gansu Academy of Agricultural Sciences.

\section{Field trial experiments and phenotype measurements}

The two populations and their parents were grown in four different locations over two years (Additional file 3). In 2015, the two populations were planted in the fields of Crop Institute of Gansu Academy of Agricultural Sciences, Lanzhou and Jingtai in Gansu Province of China, and Yuanmou in Yunnan Province. Then, the two populations were planted in the fields of Biotechnology institute of Chinese Academy of Agricultural Sciences, Langfang in Hebei Province in 2016. Three replications were included in each of the field trial experiments. Three lines including about 30 plants were included in each plot. Each plot was with $1 \mathrm{~m}$ long and $0.6 \mathrm{~m}$ wide. Five plants were randomly selected for plant height $(\mathrm{PH})$ and technical length (TL) measurements with unit $\mathrm{cm}$. The plant height was measured from the bottom to the top of the whole plant, and the technical length was referred to the length of the main stem, that mean the length from the bottom until the first branch. $\mathrm{PH}$ values were measured in all four environments, and TL values were recorded in three environments. Then the average values, standard deviation, coefficient of variation, skewness and kurtosis of each trait were analyzed by SPSS17 software.

\section{DNA extraction, library construction and sequencing}

Genomic DNA was extracted from young leaves of a single plant for each inbred line of each RIL population. The extraction method was as described by Murray and Thompson [41]. The DNA quality was checked with a Nanodrop2000 and by running it on a 1\% agarose gel.
Then, 200 ng qualified genomic DNA was used for sequence library construction and SNP marker development.

The double digested restriction site-associated DNA (ddRAD) sequencing method was used for sequence library construction and SNP marker development. ddRAD libraries for the parents and all RILs were constructed as described previously [29]. First, the $200 \mathrm{ng}$ DNA was digested with two restriction endonucleases SacI and MseI, and then adaptors with unique barcodes were added to the restriction fragments for each individual. The final ligates from 12 individuals were pooled, and then the sizes between 220 to 500 bp were separated on $2 \%$ agarose gel. Finally, the purified fragments were amplified and the sizes ranged from 270 to $550 \mathrm{bp}$ were purified with a Qiagen gel purification kit and submitted for sequencing. The sequencing was performed on Hiseq2000 platform with paired-end reads of $90 \mathrm{bp}$.

\section{SNP discovery and genotyping}

The sequencing procedure was performed when the sequence libraries were constructed.. After getting the raw data for each individual, the $5 \mathrm{bp}$ barcode and the $5 \mathrm{bp}$ on the 3' end of sequences were trimmed and the remaining 80 nucleotides of each $\mathrm{PE}$ read were kept for further analysis.

The genome-wide SNP discovery was performed using the RFAP tool pipeline, which included assembly of a pseudo-reference sequence, SNP discovery, genotyping and discrimination of allelic SNPs [29]. Next, the flanking sequences were used to perform BLAST searches against the known reference genome (https://phytozome.jgi.doe. gov/pz/portal.html) [23]. Additionally, previously published SNP sequences and SSR primer sequences from linkage maps [13, 42, 43] were collected for BLAST searches against the reference genome [23]. All of the SNP marker information was listed in Additional file 2.

\section{Individual linkage map construction and consensus linkage map integration}

When the BLAST results for SNPs collected in this study matched known SNP or SSR locus information, the SNPs could be anchored to specific LGs. After calculating the allelic SNPs, all SNP loci with less than 25\% maximum missing data and without distorted segregation by Chi-square test with 1: 1 were used for linkage map construction. The MSTMap software [44] was used to construct a high-density genetic map. All the loci were first partitioned into LGs. The major parameters for loci partitioning were as follows: distance_function, Kosambi; p_value, 0.0000001; no_map_dist, 10; missing_threshold, 0.25 ; objective_function, COUNT. After the individual linkage maps were constructed, the Mergemap software (http://alumni.cs.ucr.edu/ yonghui/mgmap.html) was used to integrate the two individual maps. 


\section{QTL mapping for plant height (PH) and technical length (TL) traits in the two populations}

QTL mapping analysis was first done for the two related populations. QTL mapping was performed using the composite interval method (CIM) with the WinQTL cartographer 2.5 software [45]. CIM was used to scan the genetic map and estimate the likelihood of a QTL and its corresponding effect every $2 \mathrm{cM}$. The forward and backward regression algorithm was used to get cofactors. After initial QTL mapping analysis, a permutation test was done to get the LOD threshold value for each QTL. After QTLs were extracted for each of the population, the QTLs were combined in the consensus linkage map. Then, the flanking sequences of all the SNP markers in the QTL confidence interval were used to blast with the flax genome sequence(https://phytozome.jgi.doe.gov/pz/ portal.html), and then the genes located in the confidence interval regions were considered as the potential candidate genes of QTLs.

\section{Additional files}

Additional file 1: The consensus linkage map from the two populations. The linkage map consisted of $15 \mathrm{LG}$. The markers in the LG with asterisk were the common markers which are used for marker integration. (PDF $2076 \mathrm{~kb}$ )

Additional file 2: SNP marker information for consensus linkage map from $\mathrm{PH}$ and $\mathrm{MH}$ populations (XLSX $712 \mathrm{~kb}$ )

Additional file 3: Detailed environmental information of the 4 locations (XLSX $9 \mathrm{~kb})$

\section{Abbreviations}

CDS: coding sequence; PCR: polymerase chain reaction; PH: plant height; QTL: quantitative trait locus; RIL: recombinant inbred line; SNP: single nucleotide polymorphism; TL: technical length

\section{Acknowledgments}

We greatly thank Dr. Ji Feng for software design of graph drawing for QTL showing. We thank Robbie Lewis, MSc, from Liwen Bianji, Edanz Group China (www.liwenbianji.cn/ac), for editing the English text of a draft of this manuscript.

\section{Funding}

This work was supported by National Natural Science Foundation of China (No.31560401) for designing of the study, including high-throughput sequencing and SNP marker design. The funding was also for writing the manuscript. The Agricultural Science and Technology Innovation Program (ASTIP) in China and Modern Agricultural Industry Technology System (CARS17-GW-04) played roles for data collection of the field trail experiments and phenotype screening, analysis the data and interpretation of these data.

\section{Availability of data and materials}

All data generated or analyzed during this study are included in this published article and its Additional files. The datasets generated and analysed during the current study are available from the corresponding author on reasonable request.

\section{Authors' contributions}

JPZ and YL carried out the SNP related works, analyzed the data and wrote the manuscript. LMW and ZD carried out the field trial experiments. TBZ and XXS participated in the field experiments. XWP and ZHD designed the experiments. All the authors have read the manuscript and approved the manuscript, and declared that they have no competing interests.

\section{Ethics approval and consent to participate}

The field trail experiments in the current study were permitted by the local government in China.

\section{Consent for publication}

Not applicable.

\section{Competing interests}

The authors declare that they have no competing interests.

\section{Publisher's Note}

Springer Nature remains neutral with regard to jurisdictional claims in published maps and institutional affiliations.

Received: 25 April 2018 Accepted: 16 July 2018

Published online: 07 August 2018

\section{References}

1. Zohary D. Monophyletic vs. polyphyletic origin of the crops on which agriculture was founded in the near east. Gen Resour Crop Evol. 1999;46(2): 133-42.

2. Lu Y. The study, evaluation and utilization on germplasm resource of Chinese flax plant. Chinas Fiber Products. 2004;5:212-6.

3. Zhang JP, Xie YP, Dang Z, Wang LM, Li WJ, Zhao W, Zhao L, Dang ZH. Oil content and fatty acid components of oilseed flax under different environments in China. Agron J. 2016;108(1):365-72.

4. Liu FH, Chen X, Long B, Shuai RY, Long CL. Historical and botanical evidence of distribution, cultivation and utilization of Linum usitatissimum L. (flax) in China. Veg Hist Archaeobot. 2011;20(6):561-6.

5. Contreras-Soto RI, Mora F, de Oliveira MAR, Higashi W, Scapim CA, Schuster I: A genome-wide association study for agronomic traits in soybean using SNP markers and SNP-based haplotype analysis. PLoS One 2017, 12(2).

6. Soto-Cerda BJ, Duguid S, Booker H, Rowland G, Diederichsen A, Cloutier S. Genomic regions underlying agronomic traits in linseed (Linum usitatissimum L.) as revealed by association mapping. J Integr Plant Biol. 2014:56(1):75-87.

7. Halbauer EM, Bohinec V, Wittenberger M, Hansel-Hohl K, Gaubitzer S, Sehr EM: Genetic diversity of flax accessions originating in the alpine region: a case study for an ex situ germplasm evaluation based on molecular marker. Euphytica 2017, 213(6)

8. Danan S, Veyrieras JB, Lefebvre V: Construction of a potato consensus map and QTL meta-analysis offer new insights into the genetic architecture of late blight resistance and plant maturity traits. BMC Plant Biol 2011, 11.

9. Chardon F, Jasinski S, Durandet M, Lecureuil A, Soulay F, Bedu M, Guerche P. Masclaux-Daubresse C. QTL meta-analysis in Arabidopsis reveals an interaction between leaf senescence and resource allocation to seeds. J Exp Bot. 2014:65(14):3949-62.

10. Daba K, Deokar A, Banniza S, Warkentin TD, Tar'an B. QTL mapping of early flowering and resistance to ascochyta blight in chickpea. Genome. 2016; 59(6):413-25.

11. Spielmeyer W, Green AG, Bittisnich D, Mendham N, Lagudah ES. Identification of quantitative trait loci contributing to fusarium wilt resistance on an AFLP linkage map of flax (Linum usitatissimum). Theor Appl Genet. 1998;97(4):633-41.

12. Oh TJ, Gorman M, Cullis CA. RFLP and RAPD mapping in flax (Linum usitatissimum). Theor Appl Genet. 2000;101(4):590-3.

13. Cloutier S, Ragupathy R, Niu ZX, Duguid S. SSR-based linkage map of flax (Linum usitatissimum L.) and mapping of QTLS underlying fatty acid composition traits. Mol Breeding. 2011;28(4):437-51.

14. Cloutier S, Ragupathy R, Miranda E, Radovanovic N, Reimer E, Walichnowski A, Ward K, Rowland G, Duguid S, Banik M. Integrated consensus genetic and physical maps of flax (Linum usitatissimum L.). Theor Appl Genet. 2012; 125(8):1783-95.

15. Soto-Cerda BJ, Duguid S, Booker H, Rowland G, Diederichsen A, Cloutier S Association mapping of seed quality traits using the Canadian flax (Linum usitatissimum L.) core collection. Theor Appl Genet. 2014;127(4):881-96.

16. Kumar S, You FM, Duguid S, Booker H, Rowland G, Cloutier S. QTL for fatty acid composition and yield in linseed (Linum usitatissimum L.). Theor Appl Genet. 2015;128(5):965-84.

17. Sudarshan GP, Kulkarni M, Akhov L, Ashe P, Shaterian H, Cloutier S, Rowland G, Wei Y, Selvaraj G. QTL mapping and molecular characterization of the 
classical D locus controlling seed and flower color in Linum usitatissimum (flax). Sci Rep. 2017;7(1):15751.

18. Liu LZ, Qu CM, Wittkop B, Yi B, Xiao Y, He YJ, Snowdon RJ, Li JN: A highdensity SNP map for accurate mapping of seed fibre QTL in Brassica napus L. PLoS One 2013, 8(12).

19. Kamfwa K, Cichy KA, Kelly JD: Genome-wide association study of agronomic traits in common bean. Plant Genome-Us 2015, 8(2).

20. Foresman BJ, Oliver RE, Jackson EW, Chao SM, Arruda MP, Kolb FL: Genomewide association mapping of barley yellow dwarf virus tolerance in spring oat (Avena sativa L.). PLoS One 2016, 11(5).

21. Kujur A, Bajaj D, Upadhyaya HD, Das S, Ranjan R, Shree T, Saxena MS, Badoni S, Kumar V, Tripathi S et al: Employing genome-wide SNP discovery and genotyping strategy to extrapolate the natural allelic diversity and domestication patterns in chickpea. Front Plant Sci 2015, 6.

22. Lu HF, Lin T, Klein J, Wang SH, Qi JJ, Zhou Q, Sun JJ, Zhang ZH, Weng YQ, Huang SW. QTL-seq identifies an early flowering QTL located near flowering locus T in cucumber. Theor Appl Genet. 2014;127(7):1491-9.

23. Wang Z, Hobson N, Galindo L, Zhu S, Shi D, McDill J, Yang L, Hawkins S, Neutelings G, Datla R, et al. The genome of flax (Linum usitatissimum) assembled de novo from short shotgun sequence reads. Plant J. 2012;72(3): 461-73.

24. van Orsouw NJ, Hogers RCJ, Janssen A, Yalcin F, Snoeijers S, Verstege E, Schneiders $\mathrm{H}$, van der Poel $\mathrm{H}$, van Oeveren J, Verstegen $\mathrm{H}$ et al: Complexity reduction of polymorphic sequences (CRoPS (TM)): a novel approach for large-scale polymorphism discovery in complex genomes. PLoS One 2007, 2(11).

25. Elshire RJ, Glaubitz JC, Sun Q, Poland JA, Kawamoto K, Buckler ES, Mitchell SE. A robust, simple genotyping-by-sequencing (GBS) approach for high diversity species. PLoS One. 2011;6(5):e19379.

26. Baird NA, Etter PD, Atwood TS, Currey MC, Shiver AL, Lewis ZA, Selker EU, Cresko WA, Johnson EA: Rapid SNP discovery and genetic mapping using sequenced RAD markers. PLoS One 2008, 3(10).

27. Miller MR, Dunham JP, Amores A, Cresko WA, Johnson EA. Rapid and costeffective polymorphism identification and genotyping using restriction site associated DNA (RAD) markers. Genome Res. 2007;17(2):240-8.

28. Sun XW, Liu DY, Zhang XF, Li WB, Liu H, Hong WG, Jiang CB, Guan N, Ma CX, Zeng HP et al: SLAF-seq: an efficient method of large-scale De novo SNP discovery and genotyping using high-throughput sequencing. PLoS One 2013, 8(3).

29. Chen X, Li XM, Zhang B, Xu JS, Wu ZK, Wang B, Li HT, Younas M, Huang L, Luo YF et al: Detection and genotyping of restriction fragment associated polymorphisms in polyploid crops with a pseudo-reference sequence: a case study in allotetraploid Brassica napus. BMC Genomics 2013, 14

30. Kumar S, You FM, Cloutier S: Genome wide SNP discovery in flax through next generation sequencing of reduced representation libraries. BMC Genomics 2012, 13.

31. Yi L, Gao F, Siqin B, Zhou Y, Li Q, Zhao X, Jia X, Zhang H. Construction of an SNP-based high-density linkage map for flax (Linum usitatissimum L.) using specific length amplified fragment sequencing (SLAF-seq) technology. PLoS One. 2017;12(12):e0189785.

32. Singh VK, Khan AW, Jaganathan D, Thudi M, Roorkiwal M, Takagi H, Garg V, Kumar V, Chitikineni A, Gaur PM, et al. QTL-seq for rapid identification of candidate genes for 100-seed weight and root/total plant dry weight ratio under rainfed conditions in chickpea. Plant Biotechnol J. 2016;14(11):2110-9.

33. Peterson BK, Weber JN, Kay EH, Fisher HS, Hoekstra HE: Double digest RADseq: an inexpensive method for De novo SNP discovery and genotyping in model and non-model species. PLoS One 2012, 7(5).

34. Poland JA, Brown PJ, Sorrells ME, Jannink JL: Development of high-density genetic maps for barley and wheat using a novel two-enzyme genotypingby-sequencing approach. PLoS One 2012, 7(2).

35. Zhou XJ, Xia YL, Ren XP, Chen YL, Huang L, Huang SM, Liao BS, Lei Y, Yan LY, Jiang HF: Construction of a SNP-based genetic linkage map in cultivated peanut based on large scale marker development using next-generation double-digest restriction-site-associated DNA sequencing (ddRADseq). BMC Genomics 2014, 15.

36. Davik J, Sargent DJ, Brurberg MB, Lien S, Kent M, Alsheikh M: A ddRAD based linkage map of the cultivated strawberry, Fragaria xananassa. PLoS One 2015, 10(9)

37. Seki M, Chono M, Nishimura T, Sato M, Yoshimura Y, Matsunaka H, Fujita M, Oda S, Kubo K, Kiribuchi-Otobe C, et al. Distribution of photoperiod- insensitive allele Ppd-A1a and its effect on heading time in Japanese wheat cultivars. Breeding Sci. 2013;63(3):309-16.

38. Duguid SD, Kenaschuk EO, Rashid KY: Macbeth flax. Canadian Journal of Plant Science 2003:83(4):803-805

39. Wang Y, Liu Y, Kang Q, Lu Y, Yang X, Guan F, Song X. The breeding report of Heiya no.14 of the new fiber flax variety. Plant Fiber and Products. 2003; 25:112-3.

40. Zhao W, Dang Z, Zhang J, Zhao L, Wang L, Dang Z. A preliminary report on introduction of 100 flax genetic resources. Gansu Agric Sci Techonol. 2011;7: $15-8$.

41. Murray MG, Thompson WF. Rapid isolation of high molecular weight plant DNA. Nucleic Acids Res. 1980;8(19):4321-5.

42. Cloutier S, Niu ZX, Datla R, Duguid S. Development and analysis of EST-SSRs for flax (Linum usitatissimum L.). Theor Appl Genet. 2009;119(1):53-63.

43. Soto-Cerda BJ, Maureira-Butler I, Munoz G, Rupayan A, Cloutier S. SSR-based population structure, molecular diversity and linkage disequilibrium analysis of a collection of flax (Linum usitatissimum L.) varying for mucilage seedcoat content. Mol Breeding. 2012;30(2):875-88.

44. Wu Y, Bhat P, Close TJ, Lonardi S: On the accurate construction of consensus genetic maps. CSB 2008 - computational systems bioinformatics conference, to appear, Stanford, Ca. 2008.

45. Wang S, Basten C, Zeng Z. Windows QTL Cartographer 2.5. Raleigh: Department of Statistics, North Carolina State University. p. 2012. ( http:// statgen.ncsu.edu/qtlcart/WQTLCart.htm)
Ready to submit your research? Choose BMC and benefit from:

- fast, convenient online submission

- thorough peer review by experienced researchers in your field

- rapid publication on acceptance

- support for research data, including large and complex data types

- gold Open Access which fosters wider collaboration and increased citations

- maximum visibility for your research: over $100 \mathrm{M}$ website views per year

At BMC, research is always in progress.

Learn more biomedcentral.com/submissions 